\title{
Prospects for the measurement of the Higgs Yukawa couplings to $b$ and $c$ quarks, and muons at CLIC
}

\author{
Christian Grefe $^{1, \mathrm{a}}$, Tomáš Laštovička ${ }^{2, \mathrm{~b}}$, Jan Strube $^{1, \mathrm{c}}$ \\ ${ }^{1}$ CERN, Geneva, Switzerland \\ ${ }^{2}$ Institute of Physics, Academy of Sciences of the Czech Republic, Prague, Czech Republic
}

Received: 14 August 2012 / Revised: 19 November 2012 / Published online: 1 February 2013

(C) The Author(s) 2013. This article is published with open access at Springerlink.com

\begin{abstract}
The investigation of the properties of the Higgs boson, especially a test of the predicted linear dependence of the branching ratios on the mass of the final state is going to be an integral part of the physics program at colliders at the energy frontier for the foreseeable future. The large Higgs boson production cross section at a $3 \mathrm{TeV}$ CLIC machine allows for a precision measurement of the Higgs branching ratios. The cross section times branching ratio of the decays $\mathrm{H} \rightarrow \mathrm{b} \overline{\mathrm{b}}, \mathrm{H} \rightarrow \mathrm{c} \overline{\mathrm{c}}$ and $\mathrm{H} \rightarrow \mu^{+} \mu^{-}$of a Standard Model Higgs boson with a mass of $120 \mathrm{GeV}$ can be measured with a statistical uncertainty of $0.23 \%, 3.1 \%$ and $15 \%$, respectively, assuming an integrated luminosity of $2 \mathrm{ab}^{-1}$.
\end{abstract}

\section{Introduction}

The Higgs mechanism of the Standard Model predicts the existence of a fundamental spin- 0 particle. Recently, the ATLAS and CMS experiments at the LHC have observed a particle which is consistent with the predictions for a Standard Model Higgs boson, but its properties remain to be studied $[1,2]$. In particular, the Standard Model predicts a linear dependence between the Higgs boson couplings to fermions and their mass. This relation could be altered by the presence of new physics. The detailed exploration of the Higgs sector is thus instrumental to our understanding of the fundamental interactions. The compact linear collider (CLIC) is a proposed $\mathrm{e}^{+} \mathrm{e}^{-}$collider with a maximum centre-ofmomentum energy $\sqrt{s}=3 \mathrm{TeV}$, based on a two-beam acceleration scheme [3]. The Higgs boson production cross section with unpolarised beams is $421 \mathrm{fb}$ in the dominant W-fusion channel. This allows for precision measurements

\footnotetext{
a e-mail: christian.grefe@cern.ch

b e-mail: tomas.lastovicka@cern.ch

c e-mail: jan.strube@cern.ch
}

of the Yukawa couplings. The beam of the $3 \mathrm{TeV}$ CLIC consists of bunch trains of 312 bunches, which are separated by $0.5 \mathrm{~ns}$. The small beam size and large electric field in the bunches, required to achieve the peak luminosity of $5.9 \times 10^{34} \mathrm{~cm}^{-2} \mathrm{~s}^{-1}$, lead to a large cross section of real and virtual two-photon processes that are a background to the processes of interest produced in the electron-positron collision. On average, real beamstrahlung photons produce $3.2 \gamma \rightarrow$ hadrons events per bunch crossing at $\sqrt{s}=3 \mathrm{TeV}$.

We present simulation studies of the measurements of the branching ratios $\mathrm{H} \rightarrow \mathrm{b} \overline{\mathrm{b}}, \mathrm{H} \rightarrow \mathrm{c} \overline{\mathrm{c}}[4]$ and $\mathrm{H} \rightarrow \mu^{+} \mu^{-}$[5] at such a machine. These studies of the Higgs branching ratios are part of the benchmarking analyses presented in the CLIC Conceptual Design Report [6]. They are carried out in a GEANT4-based simulation [7] of the CLIC_SiD [8] detector concept, with full account of Standard Model backgrounds and using a realistic reconstruction in presence of $\gamma \rightarrow$ hadrons background. The latter is reduced partly by removing hits that are out of time with the physics process, partly by advanced off-line reconstruction techniques.

\section{The CLIC_SiD detector model}

The CLIC_SiD detector model in which these studies are carried out is a general-purpose detector with a $4 \pi$ coverage and is based on the SiD concept [9] developed for the ILC. It has been adapted [8] to meet the specific detector requirements at CLIC. It is designed for particle flow calorimetry using highly granular calorimeters.

A superconducting solenoid with an inner radius of $2.7 \mathrm{~m}$ provides a central magnetic field of $5 \mathrm{~T}$. The calorimeters are placed inside the coil and consist of a 30 layer tungstensilicon electromagnetic calorimeter with $3.5 \times 3.5 \mathrm{~mm}^{2}$ segmentation, followed by a tungsten-scintillator hadronic calorimeter with 75 layers in the barrel region and a steelscintillator hadronic calorimeter with 60 layers in the endcaps. The read-out cell size in the hadronic calorimeters is 
$30 \times 30 \mathrm{~mm}^{2}$. The iron return yoke outside of the coil is instrumented with nine double-RPC layers with $30 \times 30 \mathrm{~mm}^{2}$ read-out cells for muon identification.

The silicon-only tracking system consists of five $20 \times$ $20 \mu \mathrm{m}^{2}$ pixel layers followed by five strip layers with a pitch of $25 \mu \mathrm{m}$, a read-out pitch of $50 \mu \mathrm{m}$ and a length of $92 \mathrm{~mm}$ in the barrel region. The tracking system in the endcap consists of four stereo-strip disks with similar pitch and a stereo angle of $12^{\circ}$, complemented by seven pixelated disks in the vertex and far-forward region at lower radii with pixel sizes of $20 \times 20 \mu \mathrm{m}^{2}$.

The forward region is instrumented with a LumiCal, with coverage down to $40 \mathrm{mrad}$, and a BeamCal, with coverage down to $10 \mathrm{mrad}$.

The trigger-less readout integrates over $10 \mathrm{~ns}$ for all subdetectors except the hadronic calorimeter, which has an integration time of $100 \mathrm{~ns}$ to allow for shower development in the tungsten absorber. The silicon detectors allow time stamping of the recorded hits with a precision of a few ns.

\section{Analysis framework}

The physical processes are produced with the WHIZARD $[10,11]$ event generator, taking into account the CLIC beam spectrum, with fragmentation and hadronisation handled by the Pythia [12] package. The branching ratios of a $120 \mathrm{GeV}$ Standard Model Higgs boson are: $\mathrm{BR}(\mathrm{H} \rightarrow \mathrm{b} \overline{\mathrm{b}})=$ $6.48 \times 10^{-1}, \mathrm{BR}(\mathrm{H} \rightarrow \mathrm{c} \overline{\mathrm{c}})=3.27 \times 10^{-2}$ and $\mathrm{BR}(\mathrm{H} \rightarrow$ $\left.\mu^{+} \mu^{-}\right)=2.44 \times 10^{-4}$ [13]. The events are simulated in the CLIC_SiD detector model using SLIC [14], which is a thin wrapper around GEANT4. They are reconstructed by the algorithms in the org. Icsim [15] and slicPandora [16] packages. Unlike in analyses at lower-energy linear colliders, which use DURHAM-style jet finders that operate on all particles in the event, it was found that the beam-jets of algorithms originally developed for hadron colliders, lead to a crucial improvement of the jet-energy resolution and reduce the effect of the forward-peaking $\gamma \rightarrow$ hadrons events greatly. In the analysis of Higgs decays to $b$ and c quarks, we use the $k_{t}$ algorithm [17] as implemented by the FASTJET $[18,19]$ package. The LCFI [20] package is used for flavour tagging. The assumed luminosity of the analyses is $2 \mathrm{ab}^{-1}$, corresponding to about 4 years of data taking at nominal conditions, assuming 200 days of running per year at an efficiency of $50 \%$.

\subsection{Rejection of $\gamma \rightarrow$ hadrons backgrounds}

A $3 \mathrm{TeV}$ CLIC produces $3.2 \gamma \rightarrow$ hadrons events per bunch crossing on average. The spacing of $0.5 \mathrm{~ns}$ between bunches leads to pile-up in the subdetectors, which integrate over multiple bunch crossings. Identifying the time of the physics event and reading out only a window of $10 \mathrm{~ns}$ for the subdetectors, except for the barrel of the hadronic calorimeter, for which $100 \mathrm{~ns}$ are read out, reduces the number of $\gamma \rightarrow$ hadrons events in the data sample by about a factor of 15 .

To take into account the effect of this background on the measurement, a sample of events from $\gamma \rightarrow$ hadrons corresponding to 60 bunch crossings is mixed with each physics event for the analysis of the Higgs decaying to $\mathrm{b}$ and $\mathrm{c}$ quarks. In the $\mathrm{H} \rightarrow \mu^{+} \mu^{-}$analysis, only the signal sample was mixed with events from $\gamma \rightarrow$ hadrons background. These events are also simulated in the GEANT4 model of the CLIC_SiD detector. The equivalent of 60 bunch crossings is a compromise between realistic description and computational constraints. The $\gamma \rightarrow$ hadrons events are forwardpeaking; they are described in more detail elsewhere [21]. Their contribution to hits in the barrel hadronic calorimeter, which, in principle, accumulates the equivalent of up to 200 bunch crossings, is small. Table 1 lists the physics processes that were taken into account in the analyses.

In addition to applying read-out windows off-line, the computation of the cluster time allows to further reduce this background. Assuming ns precision of the calorimeter hit times results in sub-ns precision for the cluster time, which is calculated as a truncated mean of the corresponding hit times. The production time of the reconstructed particle is obtained by correcting the cluster time for its time of flight through the magnetic field. It is required to be consistent with the start of the physics event. Consistency is defined by a time window, whose size depends on the type of particle (hadronic or electromagnetic), its momentum and polar

Table 1 List of processes considered for this analysis with their respective cross section $\sigma$ and the number of simulated events $N_{\text {events }}$. The cross section takes into account the CLIC luminosity spectrum. Cross sections marked with $*$ include a cut on the invariant mass of the muon pair to lie between 100 and $140 \mathrm{GeV}$

\begin{tabular}{lll}
\hline Process & $\sigma(\mathrm{fb})$ & Short label \\
\hline $\mathrm{e}^{+} \mathrm{e}^{-} \rightarrow \mathrm{H} v_{\mathrm{e}} \bar{v}_{\mathrm{e}} ; \mathrm{H} \rightarrow \mu^{+} \mu^{-}$ & 0.120 & $\mathrm{H} \rightarrow \mu^{+} \mu^{-}$ \\
$\mathrm{e}^{+} \mathrm{e}^{-} \rightarrow \mathrm{H} v_{\mathrm{e}} \bar{v}_{\mathrm{e}} ; \mathrm{H} \rightarrow \mathrm{b} \overline{\mathrm{b}}$ & 272 & $\mathrm{H} \rightarrow \mathrm{b} \overline{\mathrm{b}}$ \\
$\mathrm{e}^{+} \mathrm{e}^{-} \rightarrow \mathrm{H} v_{\mathrm{e}} \bar{v}_{\mathrm{e}} ; \mathrm{H} \rightarrow \mathrm{c} \overline{\mathrm{c}}$ & 13.7 & $\mathrm{H} \rightarrow \mathrm{c} \overline{\mathrm{c}}$ \\
$\mathrm{e}^{+} \mathrm{e}^{-} \rightarrow \mu^{+} \mu^{-} v \bar{v}$ & $132^{*}$ & $\mu^{+} \mu^{-} v \bar{v}$ \\
$\mathrm{e}^{+} \mathrm{e}^{-} \rightarrow \mu^{+} \mu^{-} \mathrm{e}^{+} \mathrm{e}^{-}$ & $346^{*}$ & $\mu^{+} \mu^{-} \mathrm{e}^{+} \mathrm{e}^{-}$ \\
$\mathrm{e}^{+} \mathrm{e}^{-} \rightarrow \mu^{+} \mu^{-}$ & $12^{*}$ & $\mu^{+} \mu^{-}$ \\
$\mathrm{e}^{+} \mathrm{e}^{-} \rightarrow \tau^{+} \tau^{-}$ & $250^{*}$ & $\tau^{+} \tau^{-}$ \\
$\mathrm{e}^{+} \mathrm{e}^{-} \rightarrow \tau^{+} \tau^{-} v \bar{v}$ & $125^{*}$ & $\tau^{+} \tau^{-} v \bar{v}$ \\
$\mathrm{e}^{+} \mathrm{e}^{-} \rightarrow \mathrm{q} \overline{\mathrm{q}}$ & 3100 & $\mathrm{q} \overline{\mathrm{q}}$ \\
$\mathrm{e}^{+} \mathrm{e}^{-} \rightarrow \mathrm{q} \overline{\mathrm{q}} \bar{v}$ & 1300 & $\mathrm{q} \overline{\mathrm{q}} v v$ \\
$\mathrm{e}^{+} \mathrm{e}^{-} \rightarrow \mathrm{q} \overline{\mathrm{q}} \mathrm{e}^{+} \mathrm{e}^{-}$ & 3300 & $\mathrm{q} \overline{\mathrm{q}} \mathrm{e}^{+} \mathrm{e}^{-}$ \\
$\mathrm{e}^{+} \mathrm{e}^{-} \rightarrow \mathrm{q} \overline{\mathrm{q}} \mathrm{e} \nu$ & 5300 & $\mathrm{q} \overline{\mathrm{q}} v$ \\
generator level: $\gamma \rightarrow \mu^{+} \mu^{-}$ & $20000^{*}$ & $\gamma \gamma \rightarrow \mu^{+} \mu^{-}$ \\
\hline
\end{tabular}




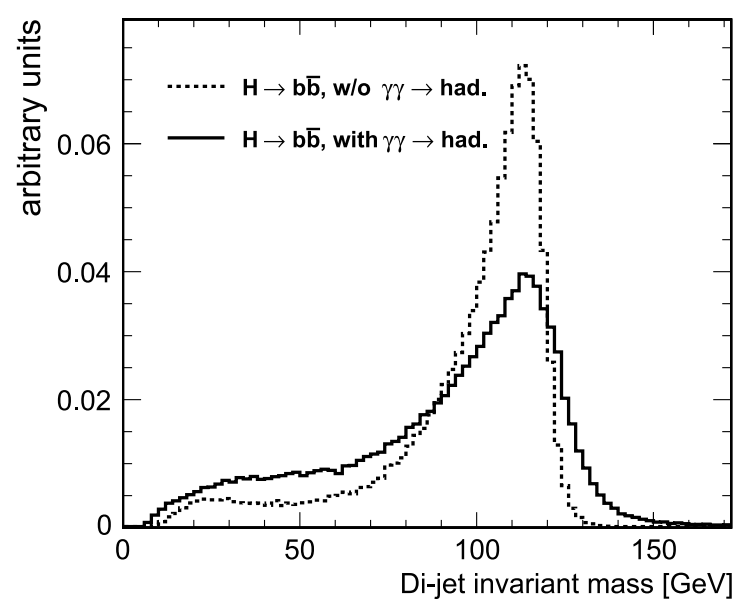

Fig. 1 Reconstructed Higgs mass spectrum in $\mathrm{H} \rightarrow \mathrm{b} \overline{\mathrm{b}}$ events without pile-up of $\gamma \rightarrow$ hadrons (dashed histogram) and with the pile-up included (solid histogram)

angle $\theta$. For example, in the $\mathrm{H} \rightarrow \mathrm{b} \overline{\mathrm{b}}$ events the fraction of energy from the $\gamma \rightarrow$ hadrons background in reconstructed jets is reduced from $22 \%$ to $6.5 \%$ by removing out-of-time particles. At the same time the reconstructed signal energy is reduced by less than $0.2 \%$.

\section{Measurement of Higgs decays to pairs of $b$ and $c$ quarks}

The particles passing the pre-selection based on the reconstructed production time are clustered into two jets using the $k_{t}$ algorithm as implemented in the FASTJET package. The size parameter $R$ for the jet clustering is 0.7 . The presence of $\gamma \rightarrow$ hadrons background affects the di-jet mass resolution of the reconstructed Higgs as shown in Fig. 1. The tail towards lower mass values is due to jets in the forward region which are only partly within the detector acceptance. The $\gamma \rightarrow$ hadrons background widens the invariant mass distribution reducing the fraction of events between $90 \mathrm{GeV}$ and $130 \mathrm{GeV}$ from $75 \%$ to $57 \%$. The peak position is shifted towards higher masses by less than $1 \mathrm{GeV}$.

The LCFI flavour tagging package finds secondary vertices in each jet and uses them, along with complementary track-based information, in a neural network to distinguish b-, c-, and light quark jets. Figure 2(a) shows the mis-tag rate for c-jets and light jets as b-jets versus the b-tag efficiency, while Fig. 2(b) shows the mis-tag rate for b-jets and light jets as c-jets versus the c-tag efficiency. The flavour tagging performance is reduced by the presence of the $\gamma \rightarrow$ hadrons background. For instance, at the b-tag efficiency of $70 \%$ the mis-tag rate for c-jets (light jets) increases from $4.3 \%$ $(0.19 \%)$ without overlay to $6.8 \%(0.33 \%)$ with overlay.

The main SM background of the measurement of the decays $\mathrm{H} \rightarrow b \bar{b}$ and $\mathrm{H} \rightarrow c \bar{c}$ is from two-jet processes $\mathrm{e}^{+} \mathrm{e}^{-} \rightarrow \mathrm{q} \overline{\mathrm{q}} \mathrm{v} \bar{v}$, due to their large cross section, and from processes with two measured jets and additional particles that escape detection. The invariant mass of the jet pair is the major discriminant between decays of Higgs and of $\mathrm{Z}$ bosons. It is used in a second neural network, together with the output of the b-flavour-tagging network and the following variables:

- The maximum of the absolute values of jet pseudorapidities.

- The sum of the remaining LCFI jet flavour tag values, i.e. c-tag against u d s b-background, c-tag against bbackground and b-tag against uds-background.

- $R_{\eta \phi}$, the distance of jets in the $\eta-\phi$ plane.

- The sum of jet energies.

- The total number of leptons in an event.

- The total number of photons in an event.

- The acoplanarity of the jets.

The neural network selection efficiency $S / S_{\text {total }}$ versus the statistical uncertainty $\sqrt{S+B} / S$ on the measurement of the number of signal events $S$ and background events $B$ is shown in Fig. 3 for the two neural networks that were trained on $\mathrm{H} \rightarrow b \bar{b}$ and $\mathrm{H} \rightarrow c \bar{c}$ as signal, respectively. The optimal selection is at the local minimum of the curve, at a selection efficiency of $52 \%$ for $\mathrm{H} \rightarrow \mathrm{b} \overline{\mathrm{b}}$ with a sample purity of $68 \%$, corresponding to a statistical uncertainty of $0.23 \%$. The optimal selection for $\mathrm{H} \rightarrow \mathrm{c} \overline{\mathrm{c}}$ has an efficiency of $24 \%$, corresponding to a sample purity of $16 \%$ and a statistical uncertainty of $3.1 \%$. Purity values reflect the fact that b-jets can be distinguished from c-jets with high purity, while incompletely reconstructed $b$-jets make up a large fraction of the background to c-jet selection, making the analysis more challenging.

\section{Measurement of Higgs decays to pairs of muons}

The measurement of the rare decay $\mathrm{H} \rightarrow \mu^{+} \mu^{-}$requires high luminosity operation and sets stringent limits on the momentum resolution of the tracking detectors. The branching ratio of the decay of a Standard Model Higgs boson to a pair of muons is important as the lower end of the accessible decays and defines the endpoint of the test of the predicted linear dependence of the branching ratios to the mass of the final state particles.

\subsection{Event selection}

The average muon reconstruction efficiency for polar angles greater than $10^{\circ}$ is $99.6 \%$ without $\gamma \rightarrow$ hadrons background. When adding this background the muon reconstruction efficiency deteriorates to $98.4 \%$ in this region of polar angles. The efficiency for smaller polar angles is limited by 
Fig. 2 Left: Efficiency of tagging a b-jet as c (dark (blue) lines) or light (light (green) lines) versus tagging it as $b$. Right: Efficiency of tagging a c-jet as b (dark (red) lines) or light (light (green) lines) versus tagging it as $\mathrm{c}$. The solid lines show performance with pile-up of $\gamma \rightarrow$ hadrons events, the dashed lines without this background

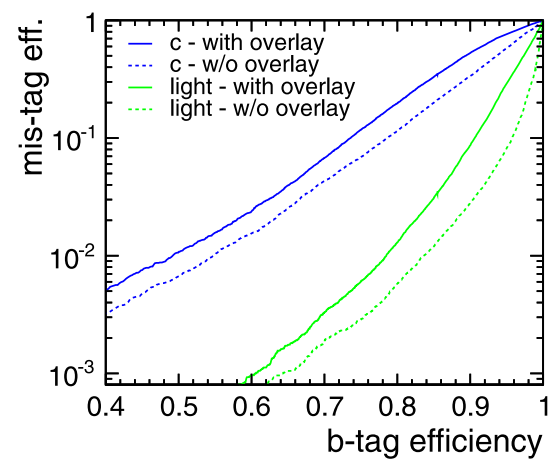

(a)

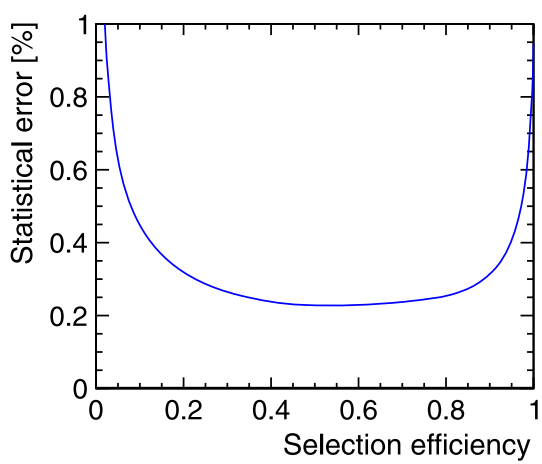

(a) $\mathrm{H} \rightarrow \mathrm{b} \overline{\mathrm{b}}$

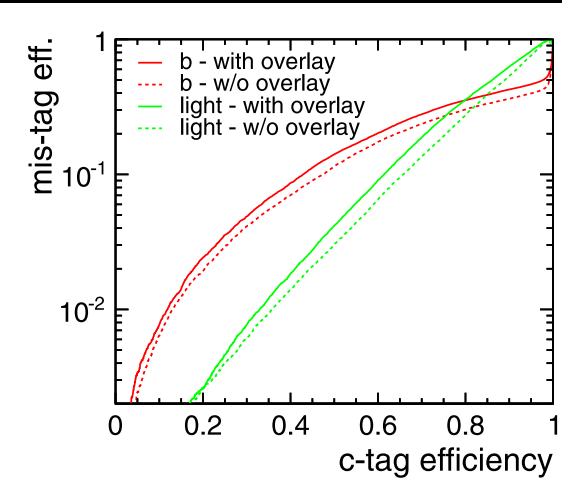

(b)

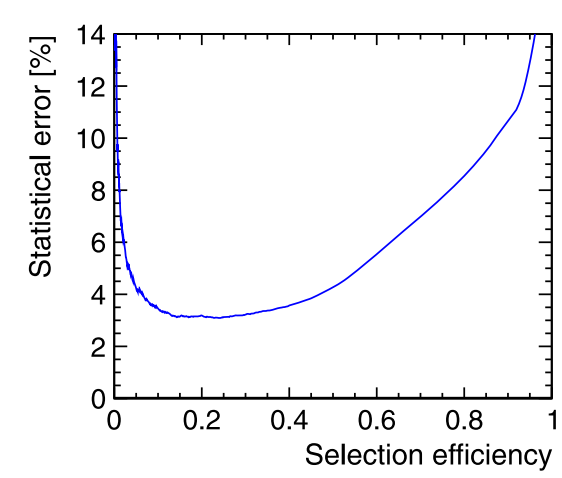

(b) $\mathrm{H} \rightarrow \mathrm{c} \overline{\mathrm{c}}$
Fig. 3 Statistical uncertainty of the measurement of cross section times branching ratio versus selection efficiency of the neural network. Left: The neural network was trained to identify $\mathrm{H} \rightarrow \mathrm{b} \overline{\mathrm{b}}$ decays from di-jet backgrounds including $\mathrm{H} \rightarrow \mathrm{c} \overline{\mathrm{c}}$. Right: The neural network was trained on $\mathrm{H} \rightarrow \mathrm{c} \overline{\mathrm{c}}$ as signal and di-jets including $\mathrm{H} \rightarrow \mathrm{b} \overline{\mathrm{b}}$ as background the acceptance of the tracking detectors. The events are required to have at least two reconstructed muons, each with a transverse momentum of more than $5 \mathrm{GeV}$. In case there are more than two muons reconstructed, the two most energetic ones are used, which are referred to as $\mu_{1}$ and $\mu_{2}$. In addition, the invariant mass of the two muons $M(\mu \mu)$ is required to be between $105 \mathrm{GeV}$ and $135 \mathrm{GeV}$. The total reconstruction efficiency of the signal sample is $72 \%$ in the presence of $\gamma \rightarrow$ hadrons background. The inefficiency is dominated by acceptance effects.

The event selection is done using the boosted decision tree (BDT) classifier implemented in TMVA [22]. The $\mu^{+} \mu^{-}, \tau^{+} \tau^{-}$and $\tau^{+} \tau^{-} \nu \bar{v}$ samples are not used in the training of the BDT, but are effectively removed by the classifier nevertheless. The variables used for the event selection by the BDT are:

- The visible energy excluding the two reconstructed muons $E_{\text {vis. }}$.

- The scalar sum of the transverse momenta of the two muons $p_{\mathrm{T}}\left(\mu_{1}\right)+p_{\mathrm{T}}\left(\mu_{2}\right)$.

- The helicity angle $\cos \theta^{*}(\mu \mu)=\frac{\mathbf{p}^{\prime}\left(\mu_{1}\right) \cdot \mathbf{p}(\mu \mu)}{\left|\mathbf{p}^{\prime}\left(\mu_{1}\right)\right| \cdot|\mathbf{p}(\mu \mu)|}$, where $\mathbf{p}^{\prime}$ is the momentum in the rest frame of the di-muon system.

- The relativistic velocity of the di-muon system $\beta(\mu \mu)$, where $\beta=\frac{v}{c}$.

- The transverse momentum of the di-muon system $p_{\mathrm{T}}(\mu \mu)$.

- The polar angle of the di-muon system $\theta(\mu \mu)$.
The most powerful variable to distinguish signal from background events is the visible energy whenever there is an electron within the detector acceptance. Otherwise the background can be reduced by the transverse momentum of the di-muon system or the sum of the two individual transverse momenta. Figure 4 clearly shows the Higgs peak in the invariant mass distribution after the event selection.

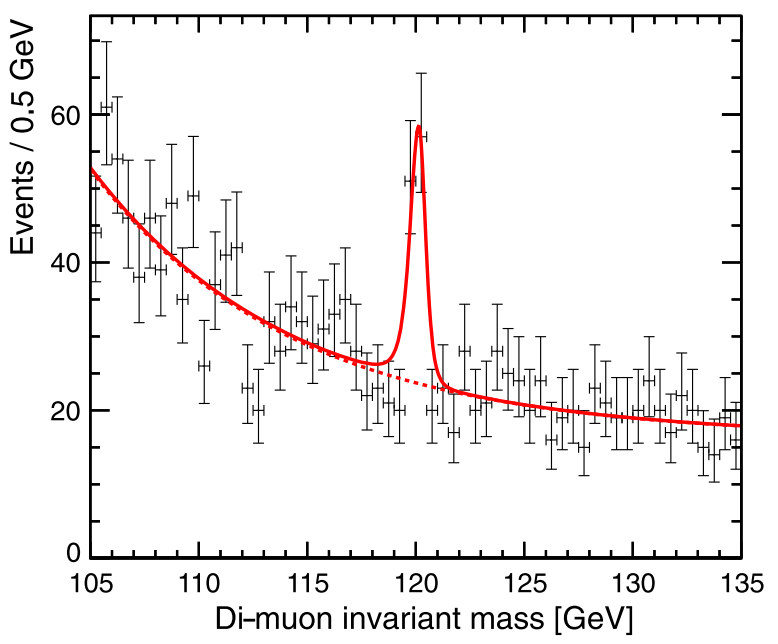

Fig. 4 Maximum likelihood fit of the Higgs mass in the data sample after selection cuts 


\subsection{Invariant mass fit}

The number of signal events is determined by an unbinned maximum likelihood fit of the invariant mass distribution of the combined signal and background sample. This sample is randomly selected from all simulated events, according to the assumed integrated luminosity of $2 \mathrm{ab}^{-1}$. The expected shapes of the signal and background contributions are determined from a fit to the full statistics of the respective sample. The distribution of the invariant mass in the $\mathrm{e}^{+} \mathrm{e}^{-} \rightarrow \mathrm{H} \rightarrow \mu^{+} \mu^{-}$sample has a tail towards lower masses because of final state radiation. It is described by two half Gaussian distributions, each with an exponential tail. The background is well described by an exponential parametrisation, obtained from a background-only sample.

The BDT selection with the highest signal significance yields a total signal selection efficiency of $21.7 \%$, corresponding to about 53 selected events in $2 \mathrm{ab}^{-1}$. The relative statistical uncertainty on the cross-section times branching ratio obtained from the fit of the invariant mass distribution is $26.3 \%$. This corresponds to a signal significance of approximately $3.8 \sigma$. Without addition of the $\gamma \gamma \rightarrow$ hadrons background the relative statistical uncertainty on the crosssection times branching ratio improves to $23 \%$, due to higher signal selection efficiency.

\subsection{Study of the momentum resolution}

The ability to measure the decay $\mathrm{H} \rightarrow \mu^{+} \mu^{-}$depends crucially on the momentum resolution of the tracking detectors. In a fast simulation study, different values for the momentum resolution were applied to the true muon momenta. For each assumed momentum resolution an individual BDT was trained to optimise the event selection for the invariant mass fit, which is performed as described above. For this study the impact of the $\gamma \gamma \rightarrow$ hadrons background was neglected. The results are shown in Table 2. We find an average resolution of at least $5 \times 10^{-5} \mathrm{GeV}^{-1}$ is required in order for the momentum resolution not to be the dominant uncertainty contribution in a $2 \mathrm{ab}^{-1}$ measurement of the decay

Table 2 Dependence of the statistical uncertainty of the measurement of cross section times branching ratio for the decay $h \rightarrow \mu^{+} \mu^{-}$on the momentum resolution $\sigma\left(\Delta p_{\mathrm{T}}\right) / p_{\mathrm{T}}^{2}$. The study assumes an integrated luminosity of $2 \mathrm{ab}^{-1}$. The values do not include the impact of the $\gamma \rightarrow$ hadrons background and the possible reduction of the $\mathrm{e}^{+} \mathrm{e}^{-} \rightarrow \mu^{+} \mu^{-}$ $\mathrm{e}^{+} \mathrm{e}^{-}$background using electron tagging in the forward calorimeters

\begin{tabular}{lll}
\hline$\sigma\left(\Delta p_{\mathrm{T}}\right) / p_{\mathrm{T}}^{2}$ & $\sigma(\Delta M(\mu \mu))$ & $\begin{array}{l}\text { Stat. } \\
\text { uncertainty }\end{array}$ \\
\hline $10^{-3} \mathrm{GeV}^{-1}$ & $6.5 \mathrm{GeV}$ & - \\
$10^{-4} \mathrm{GeV}^{-1}$ & $0.70 \mathrm{GeV}$ & $34.3 \%$ \\
$10^{-5} \mathrm{GeV}^{-1}$ & $0.068 \mathrm{GeV}$ & $18.2 \%$ \\
$10^{-6} \mathrm{GeV}^{-1}$ & $0.022 \mathrm{GeV}$ & $16.0 \%$ \\
\hline
\end{tabular}

$\mathrm{H} \rightarrow \mu^{+} \mu^{-}$. The average momentum resolution in the fully simulated $\mathrm{H} \rightarrow \mu^{+} \mu^{-}$sample is $4 \times 10^{-5} \mathrm{GeV}^{-1}$. The results from the fast simulation study are thus consistent with those found Sect. 5.2.

\subsection{Forward electron tagging}

The dominant contributions to the reducible background are from $\mathrm{Z}$ pair production, where one $\mathrm{Z}$ decays to a pair of muons and the other decays invisibly, and from the t-channel diagram contributing to $\mathrm{e}^{+} \mathrm{e}^{-} \rightarrow \mu^{+} \mu^{-} \mathrm{e}^{+} \mathrm{e}^{-}$. In the latter the electron-positron pair goes in the very forward direction. We have investigated a possible reduction of this background using the forward calorimeters LumiCal and BeamCal. The distributions of energy and angle with the outgoing beam axis of the most and second most energetic electrons in $\mathrm{e}^{+} \mathrm{e}^{-} \rightarrow \mu^{+} \mu^{-} \mathrm{e}^{+} \mathrm{e}^{-}$events are shown in Fig. 5. Although most electrons are produced at very low polar angle, a large fraction of the electrons are within the fiducial volumes of the LumiCal and BeamCal, which have an acceptance of $44 \mathrm{mrad}$ and $15 \mathrm{mrad}$, respectively, with respect to the outgoing beam axis. Since the forward calorimeters were not part of the full detector simulation, we assume ad-hoc electron tagging efficiencies in these two calorimeters to reject background events. Afterwards, a dedicated BDT classifier is trained on the pre-selected background samples using the variables described in Sect. 5.1. For example, assuming an electron tagging efficiency of $95 \%$ in the LumiCal improves the total signal selection efficiency to $49.7 \%$, which results in a statistical uncertainty on the cross-section times branching ratio measurement of $15.7 \%$. Assuming a higher electron tagging efficiency of $99 \%$ in the LumiCal improves this result to approximately $15 \%$. If the BeamCal is used in addition, assuming an average electron tagging efficiency of $70 \%$ in its fiducial volume, the statistical uncertainty can be improved to $14.5 \%$.

An independent study [23] of the electron tagging efficiency in the forward calorimeters at a CLIC detector, taking into account the $\gamma \gamma \rightarrow$ hadrons background as well as $\mathrm{e}^{+} \mathrm{e}^{-}$-pair background, confirms the efficiencies we assume here.

\section{Results}

We have demonstrated the potential of measuring the cross section times branching ratios of a $120 \mathrm{GeV}$ Higgs boson at a $3 \mathrm{TeV}$ CLIC with high precision. For the measurement of Higgs decays to quarks, $0.23 \%$ and $3.1 \%$ statistical uncertainty can be achieved for the decays $\mathrm{H} \rightarrow \mathrm{b} \overline{\mathrm{b}}$ and $\mathrm{H} \rightarrow \mathrm{c} \overline{\mathrm{c}}$, respectively. This includes the effect of background from $\gamma \rightarrow$ hadrons on the flavour tagging. Given the experience of the LEP experiments [24] in the measurements of 
Fig. 5 Kinematic distributions of the most and the second most energetic electron in $\mu^{+} \mu^{-} \mathrm{e}^{+} \mathrm{e}^{-}$ events. Left: Distribution of the simulated electron energy. Right: Distribution of the polar angle $\theta^{\prime}$ with respect to the outgoing beam axis
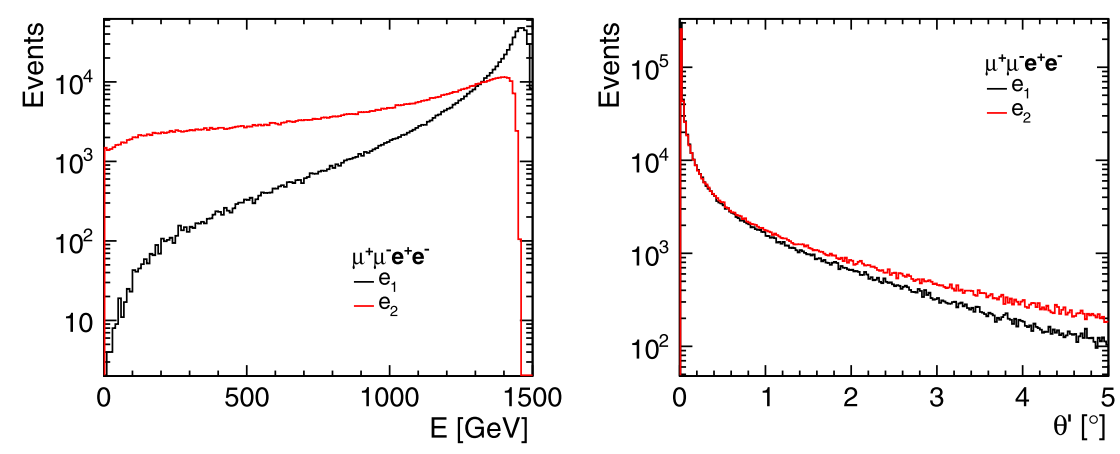

hadronic $\mathrm{Z}$ decays, with systematic uncertainties between $0.3 \%-1.2 \%$ for $R_{\mathrm{b}}^{0}$ and between $1.2 \%$ and $10 \%$ for $R_{\mathrm{c}}^{0}$, one can assume that a systematic uncertainty of around $1 \%$ is achievable in $\mathrm{H} \rightarrow \mathrm{b} \overline{\mathrm{b}}$ and around $5 \%$ in $\mathrm{H} \rightarrow \mathrm{c} \overline{\mathrm{c}}$.

For the rare decay $\mathrm{H} \rightarrow \mu^{+} \mu^{-}$, the cross section times branching ratio can be measured to a precision of about $15 \%$ if the background from $\mathrm{e}^{+} \mathrm{e}^{-} \rightarrow \mu^{+} \mu^{-} \mathrm{e}^{+} \mathrm{e}^{-}$can be reduced using tagging of electrons in the LumiCal with an efficiency of $95 \%$, and the average momentum resolution is not worse than $5 \times 10^{-5}$. The effect of background from $\gamma \rightarrow$ hadronshas been taken into account. From the measurements of the branching ratio of $\mathrm{Z}$ decays to a pair of muons at the LEP experiments, with systematic uncertainties between 0.1 and $0.4 \%$, depending on the experiment, one can assume that the systematic uncertainties related to detector effects are of the order of $1 \%$ or less. The expected uncertainty of the peak luminosity is currently being studied but is estimated to be around $1 \%$ or less.

\subsection{Extracting the Higgs coupling constants}

The uncertainties on the measurements of cross section times branching fraction can be translated to an uncertainty on the coupling constants. A global fit [25] to the complete set of measured electroweak observables gives the most accurate picture of the nature of the coupling constants. In absence of the full set of measurements, we estimate the achievable precision on the Higgs couplings in the measured channels by assuming that deviations from the Standard Model parameters occur only in the channel under consideration [26]. Using a recent overview of the uncertainties of the Standard Model Higgs branching ratios [13], Table 3 summarises conservative estimates on the achievable sensitivity to Standard Model Higgs coupling constants. For the hadronic decays, even the combination of the statistical uncertainty and a conservative average of the systematic uncertainties from similar measurements at LEP, as discussed above, is dominated by the current theoretical uncertainties of $2.8 \%$ for $\mathrm{H} \rightarrow \mathrm{b} \overline{\mathrm{b}}$ and $12.2 \%$ for $\mathrm{H} \rightarrow \mathrm{c} \overline{\mathrm{c}}$. In the case of $\mathrm{H} \rightarrow \mu^{+} \mu^{-}$the statistical uncertainties will dominate both the systematic uncertainties and the current theoretical uncertainty of $6.4 \%$.
Table 3 Statistical uncertainties of the measurements of the cross section times branching fraction, and the sensitivity to the SM b, c and $\mu$ Yukawa Higgs coupling constants at a $3 \mathrm{TeV}$ CLIC with an integrated luminosity of $2 \mathrm{ab}^{-1}$

\begin{tabular}{lll}
\hline & $\begin{array}{l}\sigma B \text { statistical } \\
\text { uncertainty (\%) }\end{array}$ & $\begin{array}{l}\text { Sensitivity to SM } \\
\text { Yukawa deviation (\%) }\end{array}$ \\
\hline $\mathrm{H} \rightarrow \mathrm{b} \overline{\mathrm{b}}$ & 0.23 & 4 \\
$\mathrm{H} \rightarrow \mathrm{c} \overline{\mathrm{c}}$ & 3.1 & 6 \\
$\mathrm{H} \rightarrow \mu^{+} \mu^{-}$ & 15 & 7.5 \\
\hline
\end{tabular}

Acknowledgements The authors would like to thank Stephane Poss for generating the event samples and managing the simulation and reconstruction on the grid.

Open Access This article is distributed under the terms of the Creative Commons Attribution License which permits any use, distribution, and reproduction in any medium, provided the original author(s) and the source are credited.

\section{References}

1. ATLAS Collaboration, Observation of a new particle in the search for the Standard Model Higgs boson with the ATLAS detector at the LHC. arXiv:1207.7214 (2012)

2. CMS Collaboration, Observation of a new boson at a mass of $125 \mathrm{GeV}$ with the CMS experiment at the LHC. arXiv:1207.7235 (2012)

3. M. Aicheler et al. (eds.), CLIC Conceptual Design Report: A Multi-TeV Linear Collider Based on CLIC Technology. CERN2012-007, Geneva (2012)

4. T. Laštovička, Light Higgs boson production and hadronic decays at 3 TeV. LCD-Note-2011-036, CERN (2011)

5. C. Grefe, Light Higgs decay into muons in the CLIC_SiD CDR detector. LCD-Note-2011-035, CERN (2011)

6. L. Linssen et al. (eds.), CLIC Conceptual Design Report: Physics and Detectors at CLIC. CERN-2012-003, Geneva (2012)

7. J. Allison et al., Geant 4 developments and applications. IEEE Trans. Nucl. Sci. 53, 270 (2006)

8. C. Grefe, A. Münnich, The CLIC_SiD_CDR geometry for the CDR Monte Carlo mass production. LCD-Note-2011-009, CERN (2011)

9. H. Aihara et al. (eds.), SiD Letter of Intent. FERMILAB-LOI2009-01 (2009)

10. W. Kilian, T. Ohl, J. Reuter, WHIZARD: simulating multi-particle processes at LHC and ILC. Eur. Phys. J. C 71, 1742 (2011) 
11. M. Moretti, T. Ohl, J. Reuter, O’Mega: an optimizing matrix element generator. arXiv:hep-ph/0102195 (2001)

12. T. Sjostrand, S. Mrenna, P.Z. Skands, PYTHIA 6.4 physics and manual. J. High Energy Phys. 0605, 026 (2006)

13. A. Denner et al., Standard model Higgs-boson branching ratios with uncertainties. Eur. Phys. J. C 71, 1753 (2011)

14. N. Graf, J. McCormick, Simulator for the linear collider (SLIC): a tool for ILC detector simulations. AIP Conf. Proc. 867, 503-512 (2006)

15. Linear collider simulations, http://lcsim.org/software/lcsim/1.18/

16. M.A. Thomson, Particle flow calorimetry and the PandoraPFA algorithm. Nucl. Instrum. Methods A 611, 25-40 (2009)

17. S.D. Ellis, D.E. Soper, Successive combination jet algorithm for hadron collisions. Phys. Rev. D 48, 3160-3166 (1993)

18. M. Cacciari, G.P. Salam, G. Soyez, FastJet user manual. Eur. Phys. J. C 72, 1896 (2012)

19. M. Cacciari, G.P. Salam, Dispelling the $N^{3}$ myth for the $k_{t}$ jetfinder. Phys. Lett. B 641, 57-61 (2006)
20. A. Bailey et al. (LCFI Collaboration), LCFIVertex package: vertexing, flavour tagging and vertex charge reconstruction with an ILC vertex detector. Nucl. Instrum. Methods Phys. Res. A 610, 573-589 (2009)

21. D. Dannheim, A. Sailer, Beam-induced backgrounds in the CLIC detectors. LCD-Note-2011-021, CERN (2011)

22. A. Höcker et al., TMVA-toolkit for multivariate data analysis. PoS ACAT, 040 (2007)

23. A. Sailer, Radiation and background levels in a CLIC detector due to beam-beam effects. PhD thesis, Humboldt-Universität zu Berlin, 2012, in preparation

24. ALEPH Collaboration, Precision electroweak measurements on the $Z$ resonance. Phys. Rep. 427, 257-454 (2006)

25. K. Desch, M. Battaglia, Determination of the Higgs profile: hfitter. AIP Conf. Proc. 578, 312-316 (2000)

26. J.D. Wells, Higgs coupling uncertainties (presentation) (2011). http://cern.ch/go/HWt7 